\title{
Longitudinal relationships among depressive symptoms and three types of memory self-report in cognitively intact older adults
}

\author{
Nikki L. Hill, ${ }^{1}$ D Jacqueline Mogle, ${ }^{2}$ Sakshi Bhargava, ${ }^{1}$ Tyler Reed Bell, ${ }^{1}$ Iris Bhang, ${ }^{1}$ \\ Mindy Katz, ${ }^{3}$ and Martin J. Sliwinski \\ ${ }^{1}$ College of Nursing, Pennsylvania State University, University Park, PA, USA \\ ${ }^{2}$ College of Health and Human Development, Pennsylvania State University, University Park, PA, USA \\ ${ }^{3}$ Saul R. Korey Department of Neurology, Albert Einstein College of Medicine, Bronx, NY, USA
}

ABSTRACT

Objectives: The current study examined whether self-reported memory problems among cognitively intact older adults changed concurrently with, preceded, or followed depressive symptoms over time.

Design: Data were collected annually via in-person comprehensive medical and neuropsychological examinations as part of the Einstein Aging Study.

Setting: Community-dwelling older adults in an urban, multi-ethnic area of New York City were interviewed.

Participants: The current study included a total of 1,162 older adults $\left(M_{\text {age }}=77.65, S D=5.03,63.39 \%\right.$ female; $74.12 \%$ White). Data were utilized from up to 11 annual waves per participant.

Measurements: Multilevel modeling tested concurrent and lagged associations between three types of memory self-report (frequency of memory problems, perceived one-year decline, and perceived ten-year decline) and depressive symptoms.

Results: Results showed that self-reported frequency of memory problems covaried with depressive symptoms only in participants who were older at baseline. Changes in perceived one-year and ten-year memory decline were related to changes in depressive symptoms across all ages. Depressive symptoms increased the likelihood of perceived ten-year memory decline the next year; however, perceived ten-year memory decline did not predict future depressive symptoms. Additionally, no significant temporal relationship was observed between depressive symptoms and self-reported frequency of memory problems or perceived one-year memory decline.

Conclusion: Our findings highlight the importance of testing the unique associations of different types of self-reported memory problems with depressive symptoms.

Key words: memory, self-report, depressive symptoms, longitudinal

\section{Introduction}

Given the insidious nature of Alzheimer's disease (AD), affected individuals often report memory changes in the years prior to diagnosis (GlodzikSobanska et al., 2007; Jessen et al., 2010), and these reports link to an increased risk of cognitive decline and $\mathrm{AD}$ many years later (Reid and Maclullich, 2006; Seo et al., 2017). Nevertheless, many self-

Correspondence should be addressed to: Nikki L. Hill, College of Nursing, Pennsylvania State University, 201 Nursing Sciences Building, University Park, PA, 16802 USA. Phone: 814-867-3265; Fax: 814-863-1027. Email: nikki.hill@psu.edu. Received 28 Jan 2019; revision requested 05 Mar 2019; revised version received 06 Jun 2019; accepted 06 Jun 2019. First published online 16 July 2019. reported memory problems are uncorroborated by objective testing (Fritsch et al., 2014; Gagnon et al., 1994; Jonker et al., 1996; Tobiansky et al., 1995), and other explanatory factors must be better understood to identify individuals at greatest risk for cognitive decline. One such factor is depressive symptoms; however, the evidence examining links between depressive symptoms and perceived memory problems is mostly cross-sectional and cannot explain how this combination occurs over time (see Hill et al., 2016 for a review). Do uncorroborated reports truly coincide with depressed mood,0. or do cross-sectional findings hide a directional influence over time? Discerning temporal relations could better inform researchers and clinicians regarding how 
reports of memory problems commonly arise, even in the absence of current cognitive impairment.

The co-occurrence of perceived memory problems and depressive symptoms is inherently complex since depression can encompass cognitive symptoms in addition to negative affect (Doumas et al., 2012; Elderkin-Thompson et al., 2011). In fact, one of the most commonly used depression measures for older adults, the Geriatric Depression Scale (GDS), includes an item that assesses selfreported memory problems: "Do you feel you have more problems with memory than most?" (Sheikh and Yesavage, 1986). Depressed older adults are more likely than their non-depressed counterparts to perceive cognitive deficits such as memory problems (Feehan et al., 1991; O'Connor et al., 1990), and the cognitive symptoms of depression can persist longer than the acute major depressive episode in older individuals (Conradi et al., 2011). Associations may represent the influence of the perception of memory problems on mood, or vice versa. The Hopelessness Theory of Depression (Abramson et al., 1989) suggests that perceptions of memory problems could precipitate depressive symptoms in older adults. This theory posits that individuals who "attribute negative life events to internal, stable, and global causes" are more likely to develop depression (Abramson et al., 1989, p. 362). For some older adults, the perception that they have memory problems may lead to worry, including concerns that they will continue to experience problems without improvement, problems will negatively impact their daily life, and problems indicate cognitive decline and possible AD development (Buckley et al., 2015). These assumptions in turn feed feelings of hopelessness about the future, leading to depressive symptoms. In line with this, Crane et al. (2007) found that the association between self-reported memory problems and depressive symptoms was mediated by negative cognitive biases such as hopelessness and low self-esteem, suggesting that in some older adults, the perception of memory problems precedes depressive symptoms due to negative cognitive biases.

The primary method for disentangling these temporal relationships is through the examination of longitudinal data linking self-reported memory problems and depressive symptoms. Some studies show that older adults who report memory problems at baseline exhibit an increased likelihood of surpassing standard cutoff scores for depression over time (Singh-Manoux et al., 2014; Tobiansky et al., 1995). For example, in one study by SinghManoux et al. (2014), older adults who reported memory problems at initial examination, compared to those who did not, had approximately twice the risk of depression (as measured by a Center for
Epidemiologic Studies Depression score of 16 or higher; Radloff, 1997) when they were reassessed after 10 years. However, the limited longitudinal evidence available provides some conflicting findings as to how self-reported memory problems alter the severity of depressive symptoms. Although Mol et al. (2009) identified elevated baseline depressive symptoms among individuals with reported memory problems compared to those without, self-reported memory problems did not escalate depressive symptoms over nine years as expected. Additional longitudinal studies examining if self-reported memory problems influence depressive symptoms over time would benefit our understanding of continuous or subsyndromal changes.

In addition to a limited amount of longitudinal research examining the relationship between selfreported memory problems and depressive symptoms over time, another limitation is that memory self-report measures vary widely across studies including the questions asked within and across measures (Rabin et al., 2015). Questions may ask respondents to rate their current memory, compare their current memory performance to past performance, or rate their memory compared to their peers. However, the influence of different measurement approaches on associations between memory self-report and outcomes such as mental and cognitive health is under-investigated (Hill et al., 2016). The lack of specificity in operationalization of the concept of self-reported memory may contribute to the inconsistencies reported across studies examining memory self-report and depressive symptoms.

Because reports of memory problems occur alongside depressive symptoms, the current study sought to investigate longitudinal relationships using three different memory self-report items. Thus, among cognitively intact older adults, we examined if self-reported memory problems changed concurrently with, preceded, or followed depressive symptoms over time. Based on previous cross-sectional work (Buckley et al., 2013; Chin et al., 2014; Hill et al., 2016; Lehrner et al., 2014), we hypothesized that, at any given year, reports of memory problems will relate to higher depressive symptoms. Secondly, grounded in the Hopelessness Theory of Depression (Abramson et al., 1989), we hypothesized that higher memory problems at a given year will predict higher depressive symptoms the subsequent year, rather than vice versa. Acknowledging the multimodal nature of subjective memory, we tested these relationships using three memory self-report items: 1) frequency of memory problems in the last year, 2) perceived one-year decline in memory, and 3) perceived ten-year decline in memory. This will inform whether specific types of self-report are 
Table 1. Inter-correlations among key study variables at baseline

\begin{tabular}{|c|c|c|c|c|c|c|c|}
\hline VARIABLE & 1 & 2 & 3 & 4 & 5 & 6 & $M(S D)$ \\
\hline 1. Age & - & & & & & & $77.647(5.032)$ \\
\hline 2. Education & $-0.090^{* * *}$ & - & & & & & $13.587(3.508)$ \\
\hline 3. Income & $-0.127^{* * *}$ & $0.348^{* * *}$ & - & & & & $2.167(0.764)$ \\
\hline 4. Frequency of Memory Problems & 0.018 & -0.012 & -0.036 & - & & & $2.656(0.722)$ \\
\hline 5. Perceived One-Year Memory Decline & 0.010 & 0.033 & 0.037 & $0.256^{* * *}$ & - & & $0.148(0.356)$ \\
\hline 6. Perceived Ten-Year Memory Decline & -0.008 & 0.031 & $0.056^{+}$ & $0.362^{* * *}$ & $0.320 * * *$ & - & $0.586(0.493)$ \\
\hline 7. Depressive Symptoms (GDS) & $0.084^{* * *}$ & $-0.158^{* * *}$ & $-0.178^{* * *}$ & 0.042 & $0.147^{* * *}$ & $0.120^{* * *}$ & $2.152(2.256)$ \\
\hline
\end{tabular}

Note: GDS $=$ Geriatric Depression Scale. ${ }^{* * *} p<.001 .{ }^{* *} p<.01 .{ }^{*} p<.05$.

differentially related to concurrent depressive symptoms and/or depressive symptoms the following year.

\section{Methods}

\section{Participants}

The study sample was drawn from the Einstein Aging Study (EAS), a longitudinal cohort study examining cognitive aging and dementia among community-dwelling older adults $(70+$ years $)$ in an urban, multi-ethnic area of New York City. EAS data were collected annually via in-person comprehensive medical and neuropsychological examinations. The study protocol was approved by the Albert Einstein College of Medicine Institutional Review Board. Full study details are described elsewhere (Katz et al., 2012). Of the 2,074 EAS participants who completed the memory self-report measures, $835(40.26 \%)$ were classified as having either amnestic mild cognitive impairment, nonamnestic mild cognitive impairment, or dementia, and thus excluded from the current study. Additionally, individuals who self-identified race/ethnicity as Asian $(n=7)$, Hispanic $(n=53)$, or other $(n=17)$ were also excluded from analyses due to the small samples they represented. The current analyses included 1,162 participants $(74.12 \%$ White, $25.88 \%$ Black; $63.39 \%$ female) who were at least 70 years old $\left(M_{\text {age }}=77.65, S D=5.03\right)$ and had no clinical diagnosis of mild cognitive impairment or dementia at any point throughout the study period. $\mathrm{Up}$ to 11 waves of data were included for each participant. At baseline, participants in the current study had an average of 13.59 years of education $(S D=3.51)$. A total of $22.23 \%$ had an annual income below $\$ 15,000$ (i.e., lived below poverty level); $38.84 \%$ of the participants' annual income was between $\$ 15,001$ and $\$ 30,000$ (i.e., lived at poverty level to up to two times above poverty level); $38.93 \%$ of the participants had an annual income above $\$ 30,000$ (i.e., lived more than two times above poverty level).

\section{Procedure}

Participants completed a neuropsychological battery as well as a detailed set of questionnaires as part of a four-hour clinic visit. The neuropsychological battery was completed approximately one to two hours prior to the questionnaires. Only questionnaire measures were used in the current analyses and are described below.

\section{Measures}

Participant characteristics and descriptive statistics for the study measures described below are provided in Table 1.

\section{DePRESSIVE SyMPTOMS}

The 15-item Geriatric Depression Scale (GDS-15; Sheikh and Yesavage, 1986) was used to measure depressive symptoms at each wave. For the current analyses, the item "Do you feel you have more problems with memory than most?" was excluded from scoring. For each of the remaining 14 items, participants responded "yes" or "no" to a series of statements, based on how they felt over the past week. Therefore, scores ranged from 0 to 14 with higher scores indicating more depressive symptoms. The GDS-15 has been found to be reliable in older adults $(\alpha=0.729$; Friedman et al., 2005) and is significantly associated with measures of depressed mood, life satisfaction, and suicidal ideation, demonstrating construct validity (Friedman et al., 2005).

MEMORY SELF-REPORT

Three memory self-report questions were administered at each wave. Frequency of memory problems in the last year was assessed with the item, "In the past year, how often did you have trouble remembering things?," with response options on a four-point scale: $1=$ never, $2=$ rarely, $3=$ sometimes, $4=$ frequently.

Perceived one-year decline in memory was assessed with the item, "Compared with one year ago, do you have trouble remembering things more often, less often, or about the same?" Perceived ten-year 
decline in memory was assessed with the item, "Compared with ten years ago, do you have trouble remembering things more often, less often, or about the same?" For both items assessing perceived decline in memory, participants responded to a three-point scale: $-1=$ less often, $0=$ about the same, $+1=$ more often. The responses were re-coded to: $0=$ less often/about the same and $1=$ more often.

\section{Statistical analysis}

We initially examined descriptive statistics and correlations in all variables required for the substantive analyses. Correlations were calculated using the Kendall Tau correction to account for the categorical nature of some variables (e.g., sex). As part of these descriptive analyses, we also examined differences in self-reported memory problems due to demographic characteristics. For these analyses, any significant effects were further probed using the Tukey's HSD to ensure a conservative approach to group comparisons as these were generally post hoc comparisons.

To address our primary research question of whether changes in self-reports of memory problems are related to changes in current and future depressive symptoms, we used separate multilevel linear models (MLM), one for each self-reported memory problem item. MLM allows the testing of longitudinal relationships among variables when there are uneven amounts of follow-up for participants as well as the separation of within-person effects (i.e., how variables change within an individual over time) from differences across individuals. Depressive symptoms and frequency of memory problems were treated as continuous outcomes and modeled using SAS proc mixed. Perceived one- and ten-year memory decline were binary variables, with response options 0 and 1 , and were modeled using SAS proc glimmix using a binary distribution with a logit link. Participants' age, sex, education, race, and income were included as covariates.

We first examined the intraclass correlations to determine the proportion of variance in each of our variables of interest that was explained by individual differences relative to change within an individual over time. Next, we fit four conditional growth models with follow-up time and our covariates as predictors to describe the trajectories of each of the three self-reported memory problems (i.e., frequency of memory problems, perceived one-year decline, and perceived ten-year decline) and depressive symptoms over time (see Table 3). Next, three substantive models examined the concurrent relationships among changes in the three self-reported memory problems and depressive symptoms. After establishing concurrent relationships, we then added changes in self-reported memory problems from the previous wave (i.e., time $t-1$ ) as a lagged variable to determine the extent to which changes in self-reports of memory problems predict future changes in depressive symptoms (i.e., time $t$ ). To fully test the temporality of these relationships, we also examined three reciprocal models in which the previous wave's depressive symptoms were used to predict future changes in the three self-reported memory problems. These additional models were necessary to understand whether there was temporal sequence of self-reported memory problems.

For all models, continuous within-person variables (e.g., frequency of memory problems) were baseline centered, that is, an individual's baseline value was subtracted from that individual's values at each wave. Both measures of perceived memory decline were entered as a raw variable as the 0 point was meaningful within as well as across individuals. The three measures of self-reported memory problems were tested as separate predictors of depressive symptoms and models were fit sequentially. Continuous between-person variables (e.g., age at baseline) were grand mean centered and appropriate reference groups were selected for included categorical variables.

\section{Results}

\section{Descriptive statistics}

At baseline, $57.29 \%$ of the participants "sometimes" experienced trouble remembering things in the past year, $7.69 \%$ "often" experienced memory problems, 27.94\% "rarely" experienced memory problems, and $7.07 \%$ "never" experienced memory problems. At baseline, $14.84 \%$ of participants reported they had trouble remembering things more often than they had one year ago. However, $58.62 \%$ had trouble remembering things more often now than they did ten years ago.

\section{INTER-CORRELATIONS}

At baseline, there were weak correlations such that participants at older ages reported more depressive symptoms $(r=.084, p<.001)$, and those more highly educated and with more income reported fewer depressive symptoms $\left(r_{\text {education }}=-.158\right.$, $\left.p<.001 ; r_{\text {income }}=-.178, p<.001\right)$. The three self-reported memory items were positively intercorrelated ( $r$ range from .256 to .362 , $p$ s $<.001$ ). There were weak positive correlations among depressive symptoms and greater perceived oneyear $(r=.147, p<.001)$ and ten-year $(r=.120$, $p<.001)$ memory decline. See Table 1 for intercorrelations and descriptive statistics. 
Table 2. Mean differences in memory self-reports and depressive symptoms by participants' sex, race, and income level at baseline

\begin{tabular}{|c|c|c|c|c|}
\hline & $\begin{array}{c}\text { FREQUENCY OF } \\
\text { MEMORY PROBLEMS }\end{array}$ & $\begin{array}{l}\text { PERCEIVED ONE-YEAR } \\
\text { MEMORY DECLINE }\end{array}$ & $\begin{array}{l}\text { PERCEIVED TEN-YEAR } \\
\text { MEMORY DECLINE }\end{array}$ & $\begin{array}{l}\text { DEPRESSIVE } \\
\text { SYMPTOMS }\end{array}$ \\
\hline \multicolumn{5}{|l|}{ Sex differences } \\
\hline Female $[\mathrm{M}(\mathrm{SD})]$ & $2.705^{*}(0.692)$ & $0.142(0.349)$ & $0.593(0.492)$ & $2.151(2.293)$ \\
\hline Male $[M(S D)]$ & $2.572^{*}(0.764)$ & $0.160(0.367)$ & $0.574(0.495)$ & $2.156(2.198)$ \\
\hline Significance Test & $\begin{array}{c}t(814.75)=2.92 \\
p<.01\end{array}$ & $\begin{array}{c}\chi^{2}(1)=0.680 \\
p=.410\end{array}$ & $\begin{array}{c}\chi^{2}(1)=0.399 \\
p=.528\end{array}$ & $\begin{array}{c}t(980)=-0.030 \\
p=.973\end{array}$ \\
\hline \multicolumn{5}{|l|}{ Race differences } \\
\hline White $[M(S D)]$ & $2.654(0.716)$ & $0.150(0.358)$ & $0.576(0.494)$ & $2.224(2.354)$ \\
\hline Black $[M(S D)]$ & $2.660(0.741)$ & $0.140(0.348)$ & $0.615(0.487)$ & $1.958(1.958)$ \\
\hline Significance Test & $\begin{array}{c}t(1127)=-0.120 \\
p=.905\end{array}$ & $\begin{array}{c}\chi^{2}(1)=0.188 \\
p=.665\end{array}$ & $\begin{array}{c}\chi^{2}(1)=1.337 \\
p=.248\end{array}$ & $\begin{array}{c}t(559.26)=1.78 \\
p=.075\end{array}$ \\
\hline \multicolumn{5}{|l|}{$\begin{array}{r}\text { Income level } \\
\text { differences }\end{array}$} \\
\hline $\begin{array}{l}\text { Below } \$ 15,000 \\
{[\mathrm{M}(\mathrm{SD})]}\end{array}$ & $2.642(0.736)$ & $0.146(0.354)$ & $0.550(0.498)$ & $3.025^{*}(2.668)$ \\
\hline $\begin{array}{l}\$ 15,000- \\
\$ 30,000 \\
{[\mathrm{M}(\mathrm{SD})]}\end{array}$ & $2.694(0.692)$ & $0.125(0.331)$ & $0.559(0.497)$ & $2.320^{*}(2.376)$ \\
\hline $\begin{array}{l}\text { Above } \$ 30,000 \\
{[M(S D)]}\end{array}$ & $2.591(0.730)$ & $0.172(0.378)$ & $0.618(0.486)$ & $1.677^{*}(1.798)$ \\
\hline Significance Test & $\begin{array}{c}F(2,1041)=2.11 \\
p=.122\end{array}$ & $\begin{array}{c}\chi^{2}(2)=3.578 \\
p=.167\end{array}$ & $\begin{array}{c}\chi^{2}(2)=4.052 \\
p=.132\end{array}$ & $\begin{array}{c}F(2,896)=22.61, \\
p<.001\end{array}$ \\
\hline \multicolumn{5}{|c|}{ Perceived one-year memory decline } \\
\hline $\begin{array}{l}\text { Better/Same } \\
{[M(S D)]}\end{array}$ & $2.574^{*}(0.714)$ & - & $0.521(0.410)$ & $2.031^{*}(2.169)$ \\
\hline Worse $[\mathrm{M}(\mathrm{SD})]$ & $3.119^{*}(0.587)$ & - & $0.964(0.186)$ & $2.986^{*}(2.584)$ \\
\hline $\begin{array}{l}\text { Significance } \\
\text { Test }\end{array}$ & $\begin{array}{c}t(261.28)=-10.72 \\
p<.001\end{array}$ & - & $\begin{array}{c}\chi^{2}(1)=115.756, \\
p<.001\end{array}$ & $\begin{array}{c}t(183.35)=-4.21, \\
p<.001\end{array}$ \\
\hline \multicolumn{5}{|c|}{ Perceived ten-year memory decline } \\
\hline $\begin{array}{c}\text { Better/Same } \\
{[\mathrm{M}(\mathrm{SD})]}\end{array}$ & $2.320^{*}(0.753)$ & & $0.013(0.113)$ & $1.825(2.042)$ \\
\hline Worse $[\mathrm{M}(\mathrm{SD})]$ & $2.891^{*}(0.597)$ & & $0.244(0.430)$ & $2.392(2.361)$ \\
\hline $\begin{array}{l}\text { Significance } \\
\text { Test }\end{array}$ & $\begin{array}{c}t(847.93)=-13.62 \\
p<.001\end{array}$ & - & $\begin{array}{c}\chi^{2}(1)=115.756, \\
p<.001\end{array}$ & $\begin{array}{c}t(867.96)=-3.94, \\
p<.001\end{array}$ \\
\hline
\end{tabular}

Note: * indicates the groups that were significantly different from each other.

BASELINE COMPARISONS

Sex differences were observed in reports of frequency of memory problems (see Table 2). At baseline, females reported significantly higher memory problem frequency $(M=2.705, S D=0.692)$ compared to males $(M=2.572, S D=0.764$; $t(814.75)=2.920, p<.01)$. Income level differences were observed in reports of depressive symptoms $(F(2,896)=22.61, p<.001)$. The Tukey HSD post hoc comparisons indicated that participants earning less than $\$ 15,000$ per year reported the most depressive symptoms $(M=3.025, S D=2.668)$, followed by participants earning between $\$ 15,001$ and $\$ 30,000(M=2.320, S D=2.376)$ and more than $\$ 30,000(M=1.677, S D=1.798)$. A chi-square test between perceived one-year and ten-year memory decline was significant $\left(\chi^{2}(1)=115.756, p<.001\right)$; at baseline, of the participants that did not perceive a change in memory over one year, $52.13 \%$ perceived a decline in their memory over ten years. However, of the participants that perceived a one-year memory decline, $96.43 \%$ also perceived a ten-year memory decline. Frequency of memory problems varied by one-year $(t(261.28)=-10.72, p<.001)$ and ten-year memory decline $(t(847.93)=-13.62$, $p<.001)$, such that participants who perceived one- and ten-year memory decline reported more frequent memory problems (one-year: $M_{\text {frequency }}=$ $3.119, S D=0.587$; ten-year: $M_{\text {frequency }}=2.891$, $S D=0.597)$ compared to their counterparts [oneyear: $\quad M_{\text {frequency }}=2.574, S D=0.714$; ten-year: $M$ frequency $=2.320, S D=0.753$ ]. Additionally, depressive symptoms also varied by one-year $[t(183.35)=-4.21, p<.001]$ and ten-year memory decline $[t(867.96)=-3.94, p<.001]$, such that participants who perceived one- and ten-year 
memory decline reported more depressive symptoms [one-year: $M_{\text {depressive }}=2.986, S D=2.584$; ten-year: $\left.\quad M_{\text {depressive }}=2.392, S D=2.361\right]$ compared to their counterparts $\left[M_{\text {depressive }}=2.031\right.$, $\left.S D=2.169 ; M_{\text {depressive }}=1.825, S D=2.042\right]$.

\section{Multilevel models}

INTRACLASS CORRELATION COEFFICIENTS (ICCS)

Prior to examining whether within-person coupling existed in participants' self-reported memory problems and depressive symptoms, ICCs were examined. The ICCs showed that $49.901 \%$ of the variation in frequency of memory problems, $47.245 \%$ of the variation in perceived one-year memory decline, $55.617 \%$ of the variation in perceived ten-year memory decline, and $77.026 \%$ of the variation in depressive symptoms was due to differences between individuals in our sample (i.e., variance explained by between-person differences rather than change within an individual over time).

\section{DESCRIPTIVE MODELS}

First, four descriptive multi-level models tested whether participants' self-reported memory problems and depressive symptoms changed over time. Participants' age, sex, education, race, and income were included as covariates (see Table 3). Average slopes indicated that for each additional year in the study, our variables of interest increased: frequency of memory problems $(b=0.012$, $S E=0.004, p<.01)$; perceived one-year memory decline (OR: 1.106; 95\% CI: 1.053 - 1.160); perceived ten-year memory decline (OR: $1.127 ; 95 \%$ CI: $1.074-1.183$ ); and depressive symptoms $(b=0.023, S E=0.010, p=.024)$.

\section{Substantive models}

Next, our substantive models simultaneously examined: 1) baseline associations of self-reported memory with depressive symptoms, 2) whether changes in participants' self-reported memory problems co-varied with changes in their depressive symptoms over time, and 3) lagged effects of self-reported memory problems on depressive symptoms and vice-versa. Three separate models examined the longitudinal associations between depressive symptoms and each of the three types of memory selfreport and the lagged effect of memory self-report on depressive symptoms (see Table 4). Additionally, three separate models examined the lagged effects of depressive symptoms on the three types of memory self-report. Age, sex, education, race, and income were included as covariates in all models (see Tables 4 and 5). Findings for the associations of depressive symptoms with self-reported memory are provided below by type of memory self-report. 
Table 4. Results of multilevel models examining co-varying and lagged relations between memory self-reports and depressive symptoms across waves: memory self-reports as predictors

OUTCOME: DEPRESSIVE SYMPTOMS

\begin{tabular}{|c|c|c|c|c|c|c|}
\hline & \multicolumn{2}{|c|}{ FREQUENCY OF MEMORY PROBLEMS } & \multicolumn{2}{|c|}{ PERCEIVED ONE-YEAR MEMORY DECLINE } & \multicolumn{2}{|c|}{ PERCEIVED TEN-YEAR MEMORY DECLINE } \\
\hline & $\begin{array}{l}\text { MODEL 1 A } \\
B(S E)\end{array}$ & $\begin{array}{l}\text { MODEL 1 B } \\
B(S E)\end{array}$ & $\begin{array}{l}\text { MODEL 2A } \\
B(S E)\end{array}$ & $\begin{array}{l}\text { MODEL 2B } \\
B(S E)\end{array}$ & $\begin{array}{c}\text { MODEL 3A } \\
B(S E)\end{array}$ & $\begin{array}{c}\text { MODEL 3B } \\
B(S E)\end{array}$ \\
\hline Intercept & $2.554^{* * *}(0.152)$ & $2.367^{* * *}(0.186)$ & $2.539^{* * *}(0.151)$ & $2.349^{* * *}(0.185)$ & $2.537^{* * *}(0.156)$ & $2.184^{* * *}(0.198)$ \\
\hline Time & $0.026^{*}(0.011)$ & $0.044^{* * *}(0.013)$ & $0.020^{+}(0.010)$ & $0.038^{* *}(0.013)$ & $0.021^{*}(0.010)$ & $0.035^{* *}(0.013)$ \\
\hline Sex $($ ref $=$ male $)$ & $-0.112(0.144)$ & $-0.227(0.173)$ & $-0.079(0.142)$ & $-0.199(0.169)$ & $-0.088(0.143)$ & $-0.198(0.174)$ \\
\hline Education (c.) & $-0.043^{*}(0.021)$ & $0.006(0.026)$ & $-0.042 *(0.021)$ & $0.006(0.025)$ & $-0.037^{+}(0.021)$ & $0.012(0.026)$ \\
\hline Age(c.) & $0.038^{* *}(0.014)$ & $0.046^{* *}(0.017)$ & $0.035^{*}(0.014)$ & $0.048^{* *}(0.017)$ & $0.020(0.016)$ & $0.032(0.020)$ \\
\hline Black (ref = White) & $-0.468^{* *}(0.158)$ & $-0.272(0.194)$ & $-0.490^{* *}(0.155)$ & $-0.316^{+}(0.189)$ & $-0.406^{* *}(0.157)$ & $-0.324^{+}(0.194)$ \\
\hline $\begin{array}{l}\text { Income }<\$ 15,000 \\
\quad(\text { ref }=\$ 15,001-\$ 30,000)\end{array}$ & $0.588^{* *}(0.193)$ & $0.410^{+}(0.246)$ & $0.549^{* *}(0.191)$ & $0.375(0.241)$ & $0.603^{* *}(0.192)$ & $0.480^{+}(0.248)$ \\
\hline $\begin{array}{l}\text { Income }>\$ 30,000 \\
\quad(\operatorname{ref}=\$ 15,001-\$ 30,000)\end{array}$ & $-0.526^{* *}(0.160)$ & $-0.516^{* *}(0.188)$ & $-0.598^{* * *}(0.158)$ & $-0.599^{* *}(0.185)$ & $-0.575^{* * *}(0.160)$ & $-0.525^{* *}(0.189)$ \\
\hline WP Effect & $0.021(0.050)$ & $0.088(0.056)$ & $0.248^{* * *}(0.075)$ & $0.302^{* * *}(0.084)$ & $0.158^{*}(0.067)$ & $0.157^{*}(0.079)$ \\
\hline WP Effect ${ }^{*}$ Age & $0.033^{* * *}(0.010)$ & $0.036^{* * *}(0.010)$ & $0.010(0.014)$ & $0.006(0.017)$ & $0.024^{+}(0.013)$ & $0.020(0.016)$ \\
\hline BP Effect(c.) & $0.241^{*}(0.096)$ & $0.390^{* *}(0.123)$ & $0.777^{* * *}(0.195)$ & $0.966^{* * *}(0.238)$ & $0.378^{* *}(0.144)$ & $0.200(0.174)$ \\
\hline Lagged Effect & - & $0.050(0.055)$ & - & $0.061(0.084)$ & - & $0.062(0.075)$ \\
\hline
\end{tabular}

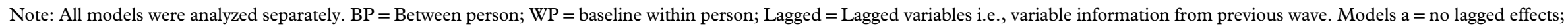
models $\mathrm{b}=$ included lagged effects; (c.) $=$ baseline values, grand mean centered.

${ }^{* * *} p<.001,{ }^{* *} p<.01,{ }^{*} p<.05 .{ }^{+} p<.10$. 
Table 5. Results of multilevel models examining co-varying and lagged relations between depressive symptoms and memory self-reports across waves: depressive symptoms as predictor

\begin{tabular}{|c|c|c|c|c|c|c|}
\hline & \multicolumn{2}{|c|}{ FREQUENCY OF MEMORY PROBLEMS } & \multicolumn{2}{|c|}{ PERCEIVED ONE-YEAR MEMORY DECLINE } & \multicolumn{2}{|c|}{ PERCEIVED TEN-YEAR MEMORY DECLINE } \\
\hline & \multicolumn{6}{|c|}{ PREDICTOR: DEPRESSIVE SYMPTOMS } \\
\hline & $\begin{array}{c}\text { MODEL } 1 \mathrm{~A} \\
B(S E)\end{array}$ & $\begin{array}{c}\text { MODEL 1 B } \\
B(S E)\end{array}$ & $\begin{array}{l}\text { MODEL 2A } \\
\text { OR }(95 \% C I)\end{array}$ & $\begin{array}{l}\text { MODEL 2B } \\
\text { OR }(95 \% \mathrm{CI})\end{array}$ & $\begin{array}{l}\text { MODEL 3A } \\
\text { OR }(95 \% \mathrm{CI})\end{array}$ & $\begin{array}{l}\text { MODEL 3B } \\
\text { OR }(95 \% \mathrm{CI})\end{array}$ \\
\hline Intercept & $2.624^{* * *}(0.046)$ & $2.630^{* * *}(0.058)$ & - & - & - & - \\
\hline Time & $0.012^{*}(0.005)$ & $0.008(0.006)$ & $1.110^{* * *}(1.054-1.169)$ & $1.062^{+}(0.997-1.131)$ & $1.103^{* * *}(1.048-1.161)$ & $1.039(0.973-1.109)$ \\
\hline Sex $(\mathrm{ref}=$ male $)$ & $0.071(0.043)$ & $0.051(0.053)$ & $1.103(0.752-1.616)$ & $1.341(0.837-2.147)$ & $1.392+(0.946-2.048)$ & $1.741^{*}(1.027-2.952)$ \\
\hline Education (c.) & $0.009(0.006)$ & $0.005(0.008)$ & $1.052^{+}(0.992-1.116)$ & $1.066^{+}(0.991-1.147)$ & $0.993(0.936-1.053)$ & $0.975(0.900-1.056)$ \\
\hline $\operatorname{Age}(\mathrm{c})$. & $-0.006(0.004)$ & $-0.010^{+}(0.005)$ & $0.978(0.941-1.016)$ & $0.952 *(0.907-0.999)$ & $0.992(0.954-1.030)$ & $0.960(0.911-1.012)$ \\
\hline Black (ref = White) & $0.015(0.048)$ & $0.002(0.060)$ & $0.845(0.546-1.309)$ & $0.713(0.411-1.238)$ & $1.214(0.788-1.869)$ & $0.944(0.520-1.715)$ \\
\hline $\begin{array}{l}\text { Income }<\$ 15,000 \\
\quad(\text { ref }=\$ 15,001-\$ 30,000)\end{array}$ & $-0.027(0.061)$ & $-0.030(0.081)$ & $1.017(0.577-1.794)$ & $0.747(0.346-1.613)$ & $0.815(0.471-1.409)$ & $0.456^{+}(0.204-1.022)$ \\
\hline $\begin{array}{l}\text { Income }>\$ 30,000 \\
\quad(\text { ref }=\$ 15,001-\$ 30,000)\end{array}$ & $-0.045(0.048)$ & $0.056(0.057)$ & $1.624^{*}(1.059-2.491)$ & $1.531(0.918-2.554)$ & $1.623^{*}(1.058-2.488)$ & $1.604(0.908-2.833)$ \\
\hline WP Effect & $0.004(0.011)$ & $0.018(0.012)$ & $1.186^{* *}(1.063-1.323)$ & $1.218^{* *}(1.080-1.375)$ & $1.138^{*}(1.016-1.275)$ & $1.106(0.968-1.264)$ \\
\hline WP Effect ${ }^{*}$ Age & $0.007^{* * *}(0.002)$ & $0.008^{* * *}(0.002)$ & $1.003(0.982-1.024)$ & $1.002(0.980-1.025)$ & $1.025^{*}(1.002-1.049)$ & $1.030 *(1.003-1.057)$ \\
\hline BP Effect (c.) & $0.028^{* *}(0.010)$ & $0.052^{* * *}(0.013)$ & $1.263^{* * *}(1.160-1.375)$ & $1.316^{* * *}(1.168-1.484)$ & $1.255^{* * *}(1.142-1.379)$ & $1.381^{* * *}(1.187-1.607)$ \\
\hline Lagged Effect & - & $0.010(0.012)$ & - & $0.977(0.858-1.114)$ & - & $1.165^{*}(1.010-1.344)$ \\
\hline
\end{tabular}

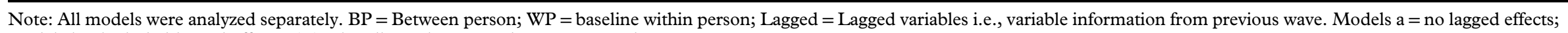
models $\mathrm{b}=$ included lagged effects; (c.) $=$ baseline values, grand mean centered.

${ }^{* * *} p<.001,{ }^{* *} p<.01,{ }^{*} p<.05 .{ }^{+} p<.10$ 
Frequency of memory problems and depressive symptoms. The model examining frequency of memory problems as the predictor of depressive symptoms (see model 1a, Table 4) showed that, after adjusting for covariates, participants who reported a higher frequency of memory problems at baseline also reported more depressive symptoms at baseline $(b=0.241, S E=0.096, p=0.013)$. Over time, change in frequency of memory problems was not significantly related to change in depressive symptoms $(b=0.021, S E=0.049, p=.671)$; however, this was qualified by a significant interaction with age at baseline $(b=0.033, S E=0.010, p<.001)$. As age was modeled continuously to probe this interaction, we computed simple slopes at values plus and minus one standard deviation in age at baseline. For participants who were older at baseline $(>\sim 83$ years), changes in self-reported frequency of memory problems were positively related to changes in depressive symptoms $(b=0.187, \quad S E=0.077$, $p=.015)$, while for participants who were younger at baseline $(<\sim 74$ years), changes in self-reported frequency of memory problems were negatively related to changes in depressive symptoms $(b=-0.145, S E=0.059, p=0.014)$. Findings for lagged effects of frequency of memory problems on depressive symptoms were not significant $(b=.050$, $S E=0.055, p=.369)$, suggesting that participants' frequency of memory problems at a given wave did not predict their depressive symptoms at the subsequent wave (see model $1 \mathrm{~b}$, Table 4 ). The model examining depressive symptoms as the predictor of frequency of memory problems showed similar findings (see model 1a, Table 5). No significant lagged effects of depressive symptoms on frequency of memory problems were found $(b=0.010$, $S E=0.012, p=.415$; see model $1 \mathrm{~b}$, Table 5 ).

Perceived one-year memory decline and depressive symptoms. The model examining perceived one-year memory decline as the predictor (see model $2 \mathrm{a}$, Table 4) showed that, at baseline, participants who reported a one-year memory decline also reported higher depressive symptoms compared to their counterparts $(b=0.777, S E=0.195, p<.001)$. Withinperson changes in perceived one-year memory decline were significantly related to changes in depressive symptoms $(b=0.248, S E=0.075, p<.010)$, such that at waves when participants perceived a one-year memory decline, they also reported more depressive symptoms. The lagged effect of perceived one-year memory decline on depressive symptoms was not significant $(b=0.061, S E=0.084, p=.466 ;$ see model $2 \mathrm{~b}$, Table 4 ).

The model examining depressive symptoms as the predictor of perceived one-year memory decline showed similar findings (see model 2a, Table 5).
Participants with one-unit higher depressive symptoms at baseline were more likely to report a oneyear decline in memory at baseline (OR: $1.263 ; 95 \%$ CI: $1.160-1.375)$ than their counterparts. Across waves, at times when participants reported a oneunit increase in depressive symptoms, they were also more likely to report a one-year decline in memory (OR: 1.186; 95\% CI: 1.063 - 1.323). The lagged effect of depressive symptoms on perceived one-year memory decline was not significant (OR: 0.977; 95\% CI: 0.858 - 1.114; see model 2b, Table 5).

Perceived ten-year memory decline and depressive symptoms. The model examining perceived ten-year memory decline as a predictor of depressive symptoms (see model 3a, Table 4) showed that, at baseline, participants' who reported a ten-year memory decline also reported more depressive symptoms compared to their counterparts $(b=0.378$, $S E=0.144, p<.01)$. Changes in perceived tenyear memory decline were significantly related to changes in depressive symptoms $(b=0.158$, $S E=0.067, p=.018)$, such that at waves when participants reported a ten-year memory decline, they also reported more depressive symptoms. The lagged effect of perceived ten-year memory decline on depressive symptoms was not significant $(b=0.062, S E=0.075, p=0.408$; see model $3 b$, Table 4).

The model examining depressive symptoms as a predictor of perceived ten-year memory decline showed similar concurrent findings but did identify a significant moderating effect of age on the association of depression with perceived ten-year memory decline across waves (see model 3a, Table 5), and a significant lagged effect of depressive symptoms on perceived ten-tear memory decline (see model 3b, Table 5). The association of depressive symptoms with perceived ten-year memory decline showed that participants with one-unit higher depressive symptoms at baseline were more likely to report a ten-year decline in memory at baseline (OR: 1.255; 95\% CI: 1.142 - 1.379). Across waves, at times when participants reported a one-unit increase in depressive symptoms, they were more likely to report a ten-year decline in memory (OR: 1.103; $95 \%$ CI: $1.048-1.161)$. This association was qualified by a significant interaction with age at baseline (OR: 1.025; 95\% CI: $1.002-1.049$ ). When participants who were older at baseline ( $>\sim 83$ years) reported a one-unit increase in depressive symptoms, they were more likely to report a ten-year decline in memory (OR: 1.288; 95\% CI: $1.073-$ 1.545). However, this association was not significant for participants who were younger at baseline $(<\sim 73$ years; OR: 1.006 ; 95\% CI: $0.876-1.157)$. Significant lagged effects of depressive symptoms on 
perceived ten-year memory were observed (OR: $1.165 ; 95 \%$ CI: $1.010-1.344$ ), such that individuals who reported a one-unit increase in depressive symptoms at a given wave were more likely to report a perceived ten-year decline at the subsequent wave.

\section{Discussion}

The purpose of the current study was to examine whether self-reported memory problems among cognitively intact older adults changed concurrently with, preceded, or followed depressive symptoms over time. We found that change in self-reported frequency of memory problems only covaried with change in depressive symptoms for the oldest individuals in our sample. That is, for individuals who were older at baseline, increases in reports of memory problem frequency were related to increases in reports of depressive symptoms. However, both one-year and ten-year perceived memory decline covaried with concurrent changes in depressive symptoms regardless of age at baseline; at waves when participants reported memory decline, regardless of the time frame for comparison, they also tended to report increases in depressive symptoms. Findings differed slightly when examining reciprocal relationships. Depressive symptoms predicted concurrent changes in self-reported memory frequency and perceived one-year memory decline regardless of age, and perceived ten-year memory decline only among those who were older at baseline. These findings support previous cross-sectional evidence linking the perception of memory problems with depressive symptoms, but also highlight the importance of the approach (i.e., specific memory selfreport items) to assess memory perceptions and their relationships with other psychosocial variables.

Although our measures of interest were concurrently associated across waves, we found only one significant lagged effect of self-reported memory on depressive symptoms - older adults' who reported depressive symptoms at a given wave were more likely to report a ten-year memory decline at the subsequent wave. Furthermore, we did not observe any significant lagged effect of self-reported memory problems on depressive symptoms. Older adults' reports of memory problems, and cognitive problems more generally, are potentially influenced by a variety of factors including age-related changes in cognitive functioning and mood disorders such as depression. Depressive symptoms are known to cooccur with self-reported memory problems, and our findings extend this body of evidence by examining these associations over time. In a recent study of middle- and older-aged adults, self-reported frequency of forgetting, seriousness of forgetting, and retrospective forgetting were associated with depressive symptoms; furthermore, individuals $\left(M_{\text {age }}=64.59, \quad S D=7.83, \quad\right.$ range $\left.=47-90\right)$ who were consistent in their reports of memory problems over a period of three years had greater depressive symptoms than those who were inconsistent in their reports or did not report memory problems over that period (Sohrabi et al., 2018). Although Sohrabi et al. did not include a measure of perceived decline to allow direct comparison with our findings, considering these longitudinal studies together suggests that aspects of the experience of memory problems - such as ratings of seriousness or decline over time are likely important to consider in future investigations of the co-occurrence of self-reported memory problems and depressive symptoms over time.

The current study demonstrates the importance of precise operationalization of the construct of selfreported memory problems; different types of memory self-report items can be differentially associated with clinically meaningful outcomes. Rabin et al. (2015) have argued that because cognitive complaints are relatively ubiquitous at older ages, asking older adults to report the number of cognitive problems experienced is less informative for identifying subtle cognitive decline than assessing other characteristics of such problems; for example, what type of memory problems they experience or whether they perceive a decline in ability over time. We found that concurrent associations between the type of memory problems reported and depressive symptoms could depend on age. There are several potential explanations for these findings. "Younger" older adults may be less sensitive, with regard to influence on depressive symptoms, to a similar frequency of memory problems compared to "older" older adults, or they may be less likely to report memory problems overall. Alternatively, younger older adults who are experiencing subtle cognitive decline may be actively adapting their activities in accordance with their abilities.

Improving our understanding of the nuances of perceived memory performance, via the precise selection of memory self-report items and interpretation of accompanying results, is critical to untangling the complex relationships among depressive and cognitive symptoms in older adults. The lack of an established standard for the assessment of perceived cognitive problems is an identified limitation in the research linking cognitive self-report with objective cognition, cognitive decline, and $\mathrm{AD}$ (Burmester et al., 2016; Crumley et al., 2014). Our findings illustrate how this limitation also contributes to our current understanding of the co-occurrence of depressive symptoms and selfreported memory problems. Across studies, the assessment of self-reported memory problems varies 
substantially, but results are typically framed as a broad concept such as "subjective memory" or "memory complaints." Two recent reviews demonstrate this extensive heterogeneity: 34 different cognitive self-report measures were used across 19 preclinical $\mathrm{AD}$ studies, and 47 different measures were used across 58 studies that examined associations between self-reported cognitive problems and affective symptoms (e.g., depressive and anxiety symptoms; Hill et al., 2016; Rabin et al., 2015). Recommendations for promoting measurement consistency have been proposed by the Subjective Cognitive Decline Initiative (SCD-I), an international working group of leading researchers in the field of preclinical AD. These include specific criteria for features of cognitive self-report that are associated with an increased likelihood of preclinical $\mathrm{AD}$, such as confirmation by an informant and perceiving that one's cognitive performance is worse than their peers. Our findings indicate that there is a similar need to identify features of cognitive/memory self-report that are most likely to be associated with concurrent affective symptoms such as depression and anxiety. Future research can help distinguish items that best discriminate between cognitive symptoms of depression, for example, and reports of cognitive problems due to other causes.

It is important to note that the measures used in this study were three single-memory self-report items, rather than validated measures as recommended by Rabin and colleagues. Relatedly, all items measured self-reported memory only, rather than other aspects of self-reported cognitive performance such as executive functioning or attention. Therefore, our findings regarding the associations of memory self-report with depressive symptoms are specific to one cognitive domain, and other aspects of self-reported cognition may demonstrate differential or stronger effects with depressive or other affective symptoms. Furthermore, both perceived decline items were dichotomously coded, which can limit their utility in longitudinal investigations. Future measurement of self-reported cognition should aim to meet current recommendations; however, previous longitudinal studies (such as EAS from which the current sample was drawn) have used more traditional, often single-item, memory measures. Although not without limitations, they do provide long periods of follow-up and an opportunity to inform future investigations using validated measures.

Although we used single-item measures, our findings suggest that considering the different aspects of self-reported memory problems (e.g., frequency vs. perceived decline) may support identification of more sensitive predictors of various outcomes of interest. Ultimately, the refinement of preclinical $\mathrm{AD}$ assessment must include the ability to discriminate self-reported cognitive problems related to affective symptoms and other factors from reports due to actual, but subtle, cognitive change.

This study explored the temporal links between self-reported memory problems and depressive symptoms in later life. Contrary to expectation, alternative cross-lagged models demonstrated few timelagged relations between perceived memory and depressive symptoms. Nonetheless, earlier depressive symptoms did account for perceived ten-year memory decline in subsequent years. Thus, it is possible that current mood might insufficiently explain longstanding perceptions of memory decline. Perhaps reflecting on broad periods like a decade prevents accurate recollection of memory errors and increases heuristic reliance on self-schemas like efficacy influenced by early periods of depressive symptomology (McAvay et al., 1996). Lack of lagged effects for other outcomes, however, could be due to the spacing of measurements (approximately one year apart). In order to identify lead-lag associations for smaller timeframes, more densely spaced repeated measures may be required. In addition, it is important to note that our sample had low levels of depressive symptoms as is common in community- and population-based samples, and relatively low levels of self-reported perceived one-year memory decline. Samples with better representation of higher levels of depression, as well as measures of perceived decline that incorporate a scale of response options targeted to severity of decline, may demonstrate associations that we were unable to detect.

For the most part, depressive symptoms and reports of memory decline co-occurred or changed together, as suggested by prior cross-sectional literature (Rami et al., 2014; Wang et al., 2000; Zlatar et al., 2017). This supports more shared than intermediary mechanisms as outlined from the Hopelessness Theory of Depression (Abramson et al., 1989). Depressive symptoms may emerge as a coinciding consequence due to the diathesis-stress process as well. Specifically, Hopelessness Theory might apply to self-reported memory problems as a related but distinct outcome in many cases. As memory errors occur over time, some older adults may perceive memory loss due to either more frequent lapses (greater stressful events) or a negative attribution bias from depressed affect that increases error awareness (stress exaggeration and potentiation); theoretically, this then precipitates hopelessness and perceived memory loss. Looking across relations, our results support a stronger tie between depressive symptoms and appraisals of decline rather than the frequency of memory problems.

Our findings have some limitations to consider. First, the current study cannot determine causal 
relationships between self-reported memory and depressive symptoms; however, longitudinal analysis provides the best method to examine the associations of interest and test directionality in observational data. Second, as previously mentioned, our measures of self-reported memory were limited by the availability of secondary data in EAS, which only allowed the use of three single memory self-report items. Examining the relationships of interest with multi-item self-reported memory measures, questions that tap into different aspects of the experience of memory problems (e.g., age-anchored comparisons or memory concerns), or questions that represent other cognitive domains may produce different findings than the present study. Also related to the self-reported items is the potentially broad interpretations by participants and bias from other recent experiences (e.g., the neuropsychological battery completed in the same visit). As noted in our previous work, the approach used in this study as well as the current literature assumes all participants are interpreting items in the same way when responding, which is an untested assumption (Hill et al., 2018). Depressive symptoms were self-reported instead of obtained from a structured clinical interview. Structured interviews align closely with diagnostic criteria while ruling out concomitant psychopathology. However, self-report from the GDS is more feasible for large epidemiological samples and quick measurement at regular clinic visits. Furthermore, the GDS retains high criterion validity with structural interviews to capture clinician-rated depressive symptoms (Wancata et al., 2006). Lastly, our sample was primarily White; several studies show disparities in which nonwhite or Hispanic older adults exhibit higher rates of depression (Dunlop et al., 2003) and cognitive decline (Sachs-Ericsson and Blazer, 2005). Future studies should purposely examine how relationships between self-reported memory and depression vary across racial-ethnic minority backgrounds with balanced samples that allow for well-powered testing. Finally, our findings provide insight into the associations of memory self-report with depressive symptoms over time, but future work should consider other self-reported cognitive problems rather than memory alone (Opdebeeck et al., 2019; Rabin et al., 2015).

Our study did have several strengths as well. First, depression was operationalized as depressive symptom count instead of odds of surpassing clinical cutoffs. While cutoffs are clinically meaningful, there is a need to understand how perceived memory problems predict depressive symptoms across the syndromic spectrum to better generalize findings to older adults in the community. Second, this study included cross-lagged relations that examined reciprocal effects of depressive symptoms on selfreported memory problems. Third, multilevel analysis allowed us to test how changes in self-reported memory problems corresponded to successive change in depressive symptoms, rather than only examining between-group differences demonstrated by previous studies (Chin et al., 2014; Fischer et al., 2008; Lehrner et al., 2014). Lastly, this study acknowledges that memory self-report is multifaceted. Future research should consider perceived memory problems as multimodal-like domains of the broader construct of memory performance; indeed, many alternative domains of memory selfreport are not yet well-examined, such as direct age-anchored comparisons (Lam et al., 2005).

\section{Conclusion}

Older adults' perceptions of memory problems, and of cognitive problems more generally, have been of increasing scientific interest over the past decade, particularly in preclinical $\mathrm{AD}$ investigations. Confounding this research, however, is the common cooccurrence of depressive symptoms, as well as a need to better understand the temporal relationships among these symptoms and self-reports of memory problems. We found that across waves, at times when older adults report perceiving memory decline, they tended to report greater depressive symptoms than at times when they did not report perceiving memory decline. Furthermore, we did not identify many temporal relationships; changes in older adults' reports of memory problems did not influence their future changes in depressive symptoms, or vice versa. However, depressive symptoms did predict greater likelihood of a perceived ten-year memory decline the next year. Future research is needed to examine whether reframing schematic sets influenced by depressive symptoms (i.e., selfefficacy) might help address concurrent memory concerns and curtail later risk for perceived or substantiated memory decline.

\section{Acknowledgments}

The authors thank the Einstein Aging Study's research team and study participants. This work was supported by National Institute on Aging, grant numbers R01AG055398 and P01 AG003949, to N.H. and R.L., respectively.

\section{Conflict of interest}

None. 


\section{Description of authors' roles}

Nikki L. Hill conceptualized the study, assisted with interpretation of results, assisted with writing the paper, and supervised the writing of the paper. Jacqueline Mogle conceptualized the study, completed the statistical analysis, and assisted with writing the paper. Sakshi Bhargava assisted with statistical analysis, interpretation of results, and writing the paper. Tyler Reed Bell assisted in the writing and final editing of the paper. Iris Bhang assisted with writing the paper. Mindy Katz supervised data collection and edited the paper. Martin J. Sliwinski conceptualized the study and provided consultation on the statistical analysis.

\section{References}

Abramson, L. Y., Metalsky, G. I. and Alloy, L. B. (1989). Hopelessness depression: a theory-based subtype of depression. Psychological Review, 96(2), 358.

Buckley, R. et al. (2013). Factors affecting subjective memory complaints in the AIBL aging study: biomarkers, memory, affect, and age. International Psychogeriatrics, 25(8), 1307-1315. doi: 10.1017/S1041610213000665.

Buckley, R.F., Saling, M. M., Frommann, I., Wolfsgruber, S. and Wagner, M. (2015). Subjective cognitive decline from a phenomenological perspective: a review of the qualitative literature. Fournal of Alzheimer's Disease, 48(Suppl. 1), S125-S140. doi: 10.3233/JAD150095.

Burmester, B., Leathem, J. and Merrick, P. (2016). Subjective cognitive complaints and objective cognitive function in aging: a systematic review and meta-analysis of recent cross-sectional findings. Neuropsychology Review, 26(4), 376-393. doi: 10.1007/s11065-016-9332-2.

Chin, J., Oh, K. J., Seo, S. W. and Na, D. L. (2014). Are depressive symptomatology and self-focused attention associated with subjective memory impairment in older adults? International Psychogeriatrics, 26(4), 573-580. doi: 10 $.1017 /$ S104161021300241X.

Conradi, H. J., Ormel, J. and de Jonge, P. (2011). Presence of individual (residual) symptoms during depressive episodes and periods of remission: a 3-year prospective study. Psychological Medicine, 41(6), 1165-1174. doi: $10.1017 /$ S0033291710001911.

Crane, M. K., Bogner, H. R., Brown, G. K. and Gallo, J. J. (2007). The link between depressive symptoms, negative cognitive bias and memory complaints in older adults. Aging and Mental Health, 11(6), 708-715. doi: 10.1080/ 13607860701368497.

Crumley, J. J., Stetler, C. A. and Horhota, M. (2014). Examining the relationship between subjective and objective memory performance in older adults: a meta-analysis. Psychology and Aging, 29(2), 250-263. doi: 10.1037/a0035908.

Doumas, M., Smolders, C., Brunfaut, E., Bouckaert, F. and Krampe, R. T. H. (2012). Dual task performance of working memory and postural control in major depressive disorder. Neuropsychology, 26(1), 110-118. doi: 10.1037/ a0026181.
Dunlop, D. D., Song, J., Lyons, J. S., Manheim, L. M. and Chang, R. W. (2003). Racial/ethnic differences in rates of depression among preretirement adults. American fournal of Public Health, 93(11), 1945-1952.

Elderkin-Thompson, V., Moody, T., Knowlton, B., Hellemann, G. and Kumar, A. (2011). Explicit and implicit memory in late-life depression. The American Fournal of Geriatric Psychiatry, 19(4), 249-255. doi: 10.1097/ JGP.0b013e3181e89a5b.

Feehan, M., Knight, R. G. and Partridge, F. M. (1991). Cognitive complaint and test performance in elderly patients suffering depression or dementia. International fournal of Geriatric Psychiatry, 6(5), 287-293. doi: 10.1002/gps .930060505.

Fischer, C. et al. (2008). Neurocognitive profiles in older adults with and without major depression. International Fournal of Geriatric Psychiatry, 23, 851-856. doi: 10.1002/ gps. 1994.

Friedman, B., Heisel, M. J. and Delavan, R. L. (2005). Psychometric properties of the 15-item geriatric depression scale in functionally impaired, cognitively intact, community-dwelling elderly primary care patients. Fournal of the American Geriatrics Society, 53, 1570-1576. doi: 10 $.1111 / j .1532-5415.2005 .53461 . x$.

Fritsch, T., McClendon, M. J., Wallendal, M. S., Hyde, T. F. and Larsen, J. D. (2014). Prevalence and cognitive bases of subjective memory complaints in older adults: evidence from a community sample. Fournal of Neurodegenerative Diseases, 2014, 1-9 doi: 10.1155/2014/ 176843.

Gagnon, M. et al. (1994). Self-reported memory complaints and memory performance in elderly French community residents: results of the PAQUID Research Program. Neuroepidemiology, 13(4), 145-154.

Glodzik-Sobanska, L. et al. (2007). Subjective memory complaints: presence, severity and future outcome in normal older subjects. Dementia and Geriatric Cognitive Disorders, 24, 177-184. doi: 10.1159/000105604.

Hill, N. L. et al. (2016). Subjective cognitive impairment and affective symptoms: a systematic review. The Gerontologist, 56(6), e109-e127. doi: 10.1093/geront/gnw091.

Hill, N. L. et al. (2018). Sources of response bias in cognitive self-report items: "Which memory are you talking about?" The Gerontologist, XX, 1-13. doi: 10.1093/geront/gny087.

Jessen, F. et al. (2010). Prediction of dementia by subjective memory impairment. Archives of General Psychiatry, 67(4), 414-422. http://dx.doi.org.ezaccess.libraries.psu.edu/10 .1001 /archgenpsychiatry.2010.30.

Jonker, C., Launer, L. J., Hooijer, C. and Lindeboom, J. (1996). Memory complaints and memory impairment in older individuals. Fournal of the American Geriatrics Society, 44(1), 44-49. doi: 10.1111/j.1532-5415.1996.tb05636.x.

Katz, M. J. et al. (2012). Age-specific and sex-specific prevalence and incidence of mild cognitive impairment, dementia, and Alzheimer dementia in blacks and whites: a report from the Einstein Aging Study. Alzheimer Disease and Associated Disorder, 26, 335-343. doi: 10.1097/WAD $.0 \mathrm{~b} 013 \mathrm{e} 31823 \mathrm{dbcfc}$.

Lam, L. C. W. et al. (2005). Subjective memory complaints in Chinese subjects with mild cognitive impairment and early Alzheimer's disease. International fournal of Geriatric Psychiatry, 20(9), 876-882. doi: 10.1002/gps.1370. 
Lehrner, J. et al. (2014). Subjective memory complaints, depressive symptoms and cognition in patients attending a memory outpatient clinic. International Psychogeriatrics, 26, 463-473. doi: 10.1017/S1041610213002263.

McAvay, G. J., Seeman, T. E., \& Rodin, J. (1996). A longitudinal study of change in domain-specific self-efficacy among older adults. The fournals of Gerontology Series B: Psychological Sciences and Social Sciences, 51(5), P243-P253.

Mol, M. E. M., van Boxtel, M. P. J., Willems, D., Verhey, F. R. J. and Jolles, J. (2009). Subjective forgetfulness is associated with lower quality of life in middle-aged and young-old individuals: a 9-year follow-up in older participants from the Maastricht Aging Study. Aging and Mental Health, 13, 699-705. http://dx.doi.org.ezaccess.libraries.psu.edu/10 $.1080 / 13607860902845541$.

O'Connor, D. W., Pollitt, P. A., Roth, M., Brook, P. B. and Reiss, B. B. (1990). Memory complaints and impairment in normal, depressed, and demented elderly persons identified in a community survey. Archives of General Psychiatry, 47(3), 224-227. doi: 10.1001/archpsyc.1990 .01810150024005 .

Opdebeeck, C., Yates, J. A., Kudlicka, A. and Martyr, A. (2019). What are subjective cognitive difficulties and do they matter? Age and Ageing, 48, 122-127. doi: 10.1093/ageing/ afy 148.

Rabin, L. A. et al. (2015). Subjective cognitive decline in older adults: an overview of self-report measures used across 19 international research studies. Fournal of Alzheimer's Disease, 48(1), S63-S86. doi: 10.3233/JAD-150154.

Radloff, L. (1997). The CES-D scale: a self report depression scale for research in the general population. Applied Psychological Measurement, 1, 385-401.

Rami, L. et al. (2014). The Subjective Cognitive Decline Questionnaire (SCD-Q): a validation study. Fournal of Alzheimer's Disease, 41, 453-466. doi: 10.3233/JAD132027.

Reid, L. M. and Maclullich, A. M. (2006). Subjective memory complaints and cognitive impairment in older people. Dementia and Geriatric Cognitive Disorders, 22, 471-485. doi: 10.1159/000096295.

Sachs-Ericsson, N. and Blazer, D. G. (2005). Racial differences in cognitive decline in a sample of community- dwelling older adults: the mediating role of education and literacy. The American fournal of Geriatric Psychiatry, 13(11), 968-975. doi: 10.1176/appi.ajgp.13.11.968.

Seo, E. H., Kim, H., Choi, K. Y., Lee, K. H. and Choo, I. H. (2017). Association of subjective memory complaint and depressive symptoms with objective cognitive functions in prodromal Alzheimer's disease including pre-mild cognitive impairment. Fournal of Affective Disorders, 217, 24-28. doi: 10.1016/j.jad.2017.03.062.

Sheikh, J. I. and Yesavage, J. A. (1986). Geriatric Depression Scale (GDS): recent evidence and development of a shorter version. Clinical Gerontologist: The fournal of Aging and Mental Health, 5(1-2), 165-173. doi: 10.1300/ J018v05n01_09

Singh-Manoux, A. et al. (2014). Subjective cognitive complaints and mortality: does the type of complaint matter? Fournal of Psychiatric Research, 48, 73-78. doi: 10.1016/j .jpsychires.2013.10.005.

Sohrabi, H. R. et al. (2018). Subjective memory complaints predict baseline but not future cognitive function over three years: results from the Western Australia Memory Study. International Psychogeriatrics, 1-13. doi: 10.1017/ S1041610218001072.

Tobiansky, R., Blizard, R., Livingston, G. and Mann, A. (1995). The Gospel Oak Study stage IV: the clinical relevance of subjective memory impairment in older people. Psychological Medicine, 25(4), 779-786.

Wancata, J., Alexandrowicz, R., Marquart, B., Weiss, M. and Friedrich, F. (2006). The criterion validity of the Geriatric Depression Scale: a systematic review. Acta Psychiatrica Scandinavica, 114(6), 398-410. doi: 10.1111/j .1600-0447.2006.00888.x.

Wang, P. N. et al. (2000). Subjective memory complaint in relation to cognitive performance and depression: a longitudinal study of a rural Chinese population. Fournal of the American Geriatrics Society, 48(3), 295-299.

Zlatar, Z. Z., Muniz, M., Galasko, D. and Salmon, D. P. (2017). Subjective cognitive decline correlates with depression symptoms and not with concurrent objective cognition in a clinic-based sample of older adults. The fournals of Gerontology. Series B, Psychological Sciences and Social Sciences. doi: 10.1093/geronb/gbw207. 